\title{
Monitoring of hydrodynamic, mechanical, geo-electrical and chemical properties of a carbonate rock during experimental dissolution
}

\author{
MARIE LEGER ${ }^{1}$, LINDA LUQUOT ${ }^{2}$ AND DELPHINE \\ ROUBINET $^{1}$ \\ ${ }^{1} \mathrm{CNRS}$ \\ ${ }^{2}$ CNRS-University of Montpellier \\ Presenting Author: marie.leger@umontpellier.fr
}

As water is an indispensable resource for human development, the characterization of geological water reservoirs is essential. Karst reservoirs are the most interesting in terms of water resources. Nevertheless, due to their high complexity, more studies are required to determine conduits and flow location. A focus on their formation during dissolution process at pore scale is needed.

For this purpose, experimental dissolutions by reactive percolation in laboratory have been done on carbonate rock samples. Cores samples with diameters of $18 \mathrm{~mm}$ and lengths of $25 \mathrm{~mm}$ were cored in a chalk block from Normandie, France. They were characterized from pore to core scale with laboratory tools, before and after their dissolution. These technics, coupled with X-ray tomography, allow to quantify hydrodynamic properties (porosity, permeability), elastic and structural properties (by acoustic and electrical measurements), pore distribution (by centrifugation). Our carbonate rocks are characterized by high porosity and an important microstructure fraction.

Samples were dissolved with fixed flow rates. Three flow rates and two acid conditions have been chosen for the samples set. Differential pressure and geo-electrical measurements have been monitoring continuously, while physico-chemical measurements were carried out from time to time. They allow to quantify hydrodynamic and structural rock changes, such as porosity and permeability, during the percolation process.

A high increase in permeability has been measured after the experiments, and preferential dissolution paths have been observed on tomographic images. Relationships between flow rates, acid concentration, percolation time, dissolved calcium concentration and stretch of path created by dissolution have been also noticed and correlated.

Dissolution of these carbonate rocks by reactive percolation allows to observe the evolution of rock parameters during a porescale karstification process, and thus better understand the location of conduits and flows in karstic reservoirs. 